\title{
Orientation and Geoeffectiveness of Magnetic Clouds as Consequences of Filament Eruptions
}

\author{
Yuming Wang ${ }^{1}$, Guiping Zhou ${ }^{2}$, Pinzhong Ye ${ }^{1}$, S. Wang ${ }^{1}$ \\ and Jingxiu Wang ${ }^{2}$ \\ ${ }^{1}$ School of Earth \& Space Sci., Univ. of Sci. \& Tech. of China, Hefei, Anhui 230026, China. \\ email: ymwang@ustc.edu.cn \\ ${ }^{2}$ National Astronomical Observatories of China.
}

\begin{abstract}
By investigating ten typical magnetic clouds (MCs) associated with large geomagnetic storms (Dst $\leqslant-100 \mathrm{nT}$ ) from 2000 to 2003, the geoeffectiveness of MCs with various orientations is addressed. It is found that the Dst peak values during the geomagnetic storms are well estimated by applying flux rope model to these magnetic clouds. A high correlation between estimated and observed Dst values is obtained. Moreover, the effect of orientations of MCs on intensities of geomagnetic storms is studied. It is found that the favorable orientations of MCs are approximately at $\theta \sim 70^{\circ}$ and $\phi \sim 40^{\circ}$ in GSE coordinates to cause large geomagnetic storms. Further, by analyzing solar observations of four associated erupted filaments, the question who determine the orientations of MCs is studied. The likelihood of predicting the intensities of a geomagnetic storms several tens hours before their occurrences is also discussed.
\end{abstract}

Keywords. Sun: coronal mass ejections (CMEs), filament, magnetic fields, solar-terrestrial relations

\section{Introduction}

Geomagnetic storm is one of most important aspect in effecting the environment around the Earth. Large non-recurrent geomagnetic storms are usually caused by interplanetary ejecta, especially magnetic clouds (MCs), and shock sheaths preceding them (e.g., Sheeley, Jr. et al. 1985; Gosling et al. 1991). These notable perturbations change some properties of interplanetary medium greatly. The relationship between interplanetary parameters and intensities of geomagnetic storms are studied exhaustively in the past several decades (e.g., Burton et al. 1975; Gonzalez et al. 1989; Vassiliadis et al. 1999; Wang et al. 2003). Fast solar wind $(V)$, strong southward component $\left(B_{s}\right)$ of magnetic fields and long duration of $B_{s}(\Delta t)$ are the most pivotal to create large geomagnetic storms (e.g., Gonzalez et al. 1994).

Intensity of a geomagnetic storm may be quantified by Dst indicator. Recent work suggested that there is a high correlation of $V B_{s}, \Delta t$ (duration of $B_{s}$ ) with Dst peak values (Wang et al. 2003). Although a good estimation of Dst storm intensity can be excepted based on the observations of interplanetary medium, there is only rough an hour before arrival of peak of geomagnetic storm, i.e., only an almost real-time prediction can be made. Gonzalez et al. (2004) proposed recently that projected speeds of halo CMEs may be used to estimate Dst peak values. But the accuracy is bad (Kane 2004). How to predict intensity of a Dst storm several ten hours before its occurrence is an important and interesting topic in space weather research. This paper aims at it, and puts emphasis on filament-associated magnetic clouds, one of main source of large geomagnetic storms. 
Table 1. List of typical magnetic clouds during $2000-2003$

\begin{tabular}{|c|c|c|c|c|c|c|c|c|c|c|c|c|c|c|c|c|c|}
\hline \multirow{2}{*}{ No. } & \multirow{2}{*}{ Date } & \multicolumn{3}{|c|}{ | Observations } & \multicolumn{8}{|c|}{ Fitted parameters } & \multirow{2}{*}{ Dst } & \multirow{2}{*}{\multicolumn{3}{|c|}{$\begin{array}{l}\text { Associated filaments } \\
\text { Date Location Tilt }\end{array}$}} & \multirow{2}{*}{$\Delta \alpha^{l}$} \\
\hline & & $V_{0}^{a}$ & $B$ & $B_{s}^{b}$ & $B_{0}^{c}$ & $H^{d}$ & $\theta^{e}$ & $\phi^{f}$ & $R^{g}$ & $D^{h}$ & $\chi^{2} / \mathrm{cc}^{i}$ & $\mathrm{Dst}^{j}$ & & & & & \\
\hline 1 & $2000.7 .15-16$ & 960 & 55 & 54 & $\mid 45.5$ & -1 & 0.2 & 70 & 7.9 & 0.051 & $0.065 / 0.92$ & -361 & $|-301|$ & \begin{tabular}{|l|l|}
7.14 \\
\end{tabular} & N17W02 & $0^{\circ}$ & $0^{\circ}$ \\
\hline 2 & 2000.8.10-11 & 440 & 14 & 13 & 11.5 & -1 & -44 & 3 & 19.2 & 0.417 & $0.057 / 0.97$ & -79 & -106 & & & & \\
\hline 3 & $2000.8 .12-13$ & 630 & 34 & 30 & 35.9 & -1 & -33 & 115 & 11.5 & 0.174 & $0.069 / 0.94$ & -248 & -235 & 8.9 & N20E15 & $45^{\circ}$ & $9^{\circ}$ \\
\hline 4 & $2000.10 .28-29$ & 400 & 20 & 18 & 18.3 & -1 & -57 & 187 & 20.8 & 0.168 & $0.017 / 0.99$ & -105 & -127 & & & & \\
\hline 5 & 2000.11.6-7 & 550 & 25 & 16 & 24.5 & -1 & -7 & 118 & 10.6 & 0.217 & $0.037 / 0.97$ & -117 & -159 & & & & \\
\hline 6 & 2001.3.19-21 & 400 & 22 & 21 & 26.0 & -1 & -72 & 286 & 20.5 & 0.459 & $0.032 / 0.96$ & -135 & -149 & & & & \\
\hline 7 & $2001.4 .21-23$ & 370 & 16 & 14 & 14.0 & -1 & -45 & 289 & 11.5 & 0.261 & $0.036 / 0.96$ & -66 & -102 & & & & \\
\hline 8 & 2002.4.17-19 & 500 & 15 & 13 & 12.0 & 1 & -54 & 163 & 20.4 & 0.181 & $0.069 / 0.93$ & -89 & -127 & & & & \\
\hline 9 & $2003.10 .29-30$ & $1500^{*}$ & 49 & 30 & 52.5 & -1 & -12 & 246 & 6.5 & 0.508 & $0.038 / 0.95$ & -156 & -363 & 10.28 & S16E15 & $-30^{\circ}$ & $17^{\circ}$ \\
\hline 10 & $2003.11 .20-21$ & 640 & 56 & 54 & 48.0 & 1 & -51 & 90 & 6.8 & 0.059 & $0.057 / 0.95$ & -421 & -472 & 11.18 & N03E18 & $0^{\circ}$ & $51^{\circ}$ \\
\hline
\end{tabular}

${ }^{a}$ Center speed of magnetic cloud. ${ }^{b}$ Maximum of southward component of magnetic field inside magnetic cloud. ${ }^{c}$ Magnetic field magnitude at the axis of flux rope. ${ }^{d}$ Sign of helicity of flux rope. ${ }^{e}$ Elevation angle of axial field (i.e., axis) of flux rope in GSE coordinates. ${ }^{f}$ Azimuthal angle of axial field (i.e., axis) of flux rope in GSE coordinates. ${ }^{g}$ Radius of flux rope. ${ }^{h}$ Distance of the closest approaching to flux rope. ${ }^{i}$ Goodness of fit. ${ }^{j}$ Estimated Dst peak value. ${ }^{k}$ Tilt of filament to solar equator. ${ }^{l}$ Angle projected in the plane perpendicular to the Sun-Earth line between filament and magnetic cloud. ${ }^{*}$ The speed is not reliable.
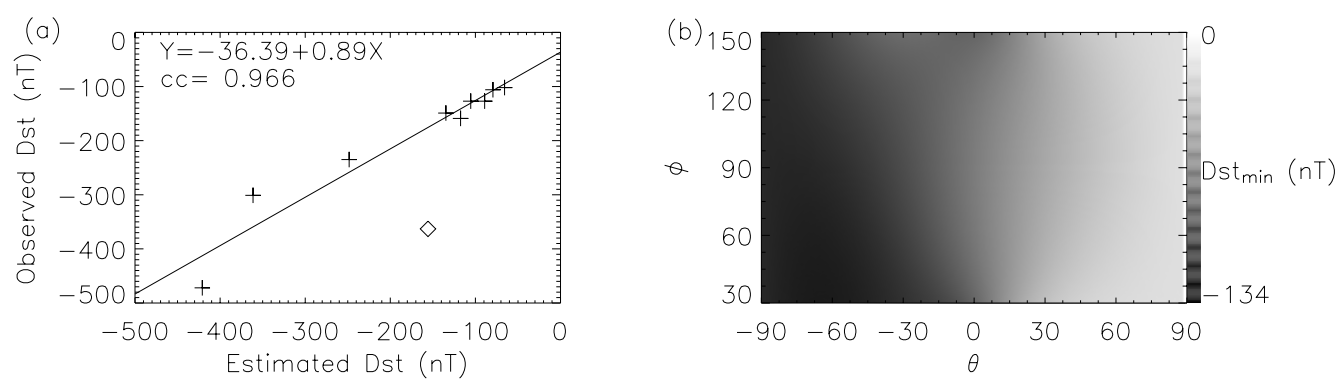

Figure 1. The left panel shows the correlation between the estimated and observed Dst peak values. The right panel exhibits Dst as a function of the elevation $\theta$ and azimuthal $\phi$ of the axis of a given cloud ( $B_{0}=20 \mathrm{nT}, R=12$ hours $D=0.2 R$, and $V=450 \mathrm{~km} / \mathrm{s}$ ) in GSE coordinates.

\section{Geoeffectiveness}

Generally, MCs can be modeled by flux ropes (e.g., Burlaga 1988; Kumar and Rust 1996). It is obvious that $B_{s}$ and $\Delta t$, the pivotal factors of causing geomagnetic storms, are dependent on the orientation of MC's axis for a magnetic cloud in consideration of flux rope model. Therefore, Zhao (2002) suggested that the orientation of MC is probably an important factor in geomagnetic storm. How good is the correlation between orientations of MCs and intensities of Dst storms? We check the MC-associated geomagnetic storms during 2000 to 2003. In selection of events, the following conditions are applied: (1) it was a large storm, i.e., Dst $\leqslant-100 \mathrm{nT} ;(2)$ the associated MC was typical. Ten events are chose. Table 1 lists the observations of them as well as some fitted parameters by using force-free flux rope model. The goodness of fit of these clouds are all high as indicated by $\chi^{2}$ and cc (correlation coefficient) listed in the 12th column. It is suggested that flux rope is a very close approximation to these typical MCs.

Wang et al. (2003) ever found an empirical formula to estimate Dst peak value during a geomagnetic storm. It is described as $D s t_{\min }=-19.01-8.43\left(-\overline{V B_{z}}\right)^{1.09}(\Delta t)^{0.30} \mathrm{nT}$. Based on the flux rope model, the values of $B_{s}$ and $\Delta t$ needed as input parameters by above formula may be derived from the fitted parameters of MCs, and therefore the Dst peak values can be estimated.

Figure 1(a) exhibits the result how consistent the estimated Dst values are with the observed values. Since the solar wind data was not reliable during the October 29, 2003 
event and the speed of that MC is therefore uncertain, the October 29, 2003 event is excluded in our fitting procedure. It is obvious that except the October 29, 2003 event marked by the diamond in Fig. 1(a), all points are near the linear-fitting line. The correlation coefficient reaches 0.97 . It implies that the flux rope model can be used to well predict the intensities of geomagnetic storms for typical magnetic clouds.

Further, for a magnetic cloud with given values of $B_{0}=20 \mathrm{nT}, R=12$ hours, $D=$ $0.2 R$, and $V_{0}=450 \mathrm{~km} / \mathrm{s}$, the estimated Dst peak values as a function of the orientations of this cloud is shown in Figure 1(b). The situation in the region of $180^{\circ} \leqslant \phi<360^{\circ}$ is not represented, because it is the same as that in the region of $0^{\circ} \leqslant \phi<180^{\circ}$. Moreover, if $\phi$ approaches to $0^{\circ}$ or $180^{\circ}$, i.e., the orientation is roughly parallel to the Sun-Earth line, $\Delta t$ will become very long that is unreasonable. So the regions of $\left[0^{\circ}, 30^{\circ}\right]$ and $\left[150^{\circ}, 180^{\circ}\right]$ are also not plotted. From Fig 1(b), it is found that intense geomagnetic storms mainly concentrate in the negative $\theta$, and the variation of storm's intensity is more sensitive in $\theta$ than in $\phi$. The most favorable orientations of MCs to cause large geomagnetic storms are at $\theta \sim-70^{\circ}$ and $\phi \sim 40^{\circ}$.

\section{Orientation}

The last section suggests that the orientations of MCs do play an important role, and the prediction of intensities of geomagnetic storms by applying force-free flux rope model is feasible. Then, who determine orientations of MCs? Lots of previous work suggested that, for filament-associated magnetic clouds, their orientations are consistent with the directions of associated filaments (e.g., Bothmer and Schwenn 1994; Marubashi 1997; McAllister et al. 2001; Yurchyshyn et al. 2001). We address this problem again by analyzing the above events. There are only four MCs associated with obvious filament eruptions among all the ten events. The last four columns in Table 1 list them.

July 14, 2000 event. Figure 2(a) exhibits this event. The left upper panel is the EIT195Aimage showing the post-flare loops overlying the erupted filament. The right upper image is the photospheric magnetic field observed by MDI/SOHO. White denotes positive polarity and black denotes negative polarity. The thick line means the filament and the thin lines from positive polarity to negative polarity indicate the arcades overlying the filament. As a comparison, the sketch of the fitted interplanetary cloud is plotted at right lower corner, in which the thick arrow denotes the axial magnetic field, i.e., the orientation, and the thin arrow indicates the ring field, i.e., the rotation of magnetic field inside the cloud. The left lower picture shows a $3-\mathrm{D}$ view of the $\mathrm{MC}$ and the associated filament. It is evident that the orientation of $\mathrm{MC}$ is very consistent with the direction of the filament $\left(\Delta \alpha \sim 0^{\circ}\right)$. The direction of the ring field of the cloud is also consistent with the direction of the arcades overlying the filament.

August 9, 2000 event. Figure 2(b) exhibits this event. Like the previous one, the orientation and the ring field of the cloud are both consistent with the solar observations of the erupted filament.

October 28, 2003 event. Figure 2(c) exhibits this event. Since this event is complicated, more observations are used. The upper middle image is obtained from Trace spacecraft, and the upper right image is the $H_{\alpha}$ observations overlapped by MDI observations. The orientation and ring field also primarily follow the erupted filament.

November 18, 2003 event. Figure 2(d) exhibits this event. The direction of the ring field of this magnetic cloud is roughly consistent with the direction of arcades overlying the erupted filament. Nevertheless, the orientation of the cloud deviates largely from the direction of the filament. The $\Delta \alpha$ reaches about $51^{\circ}$ as shown in the left lower 3-D view. 


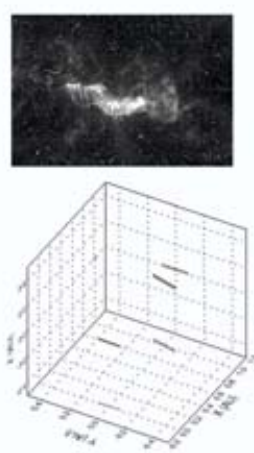

Event 1, 2000.7.14

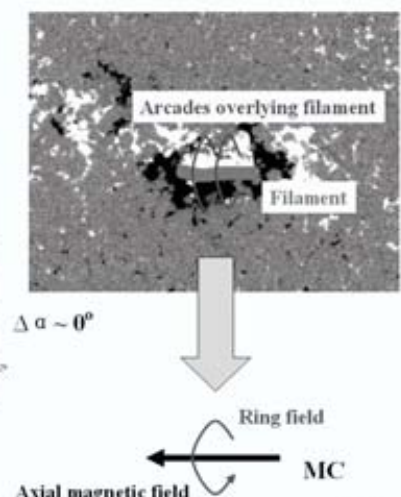

(a)

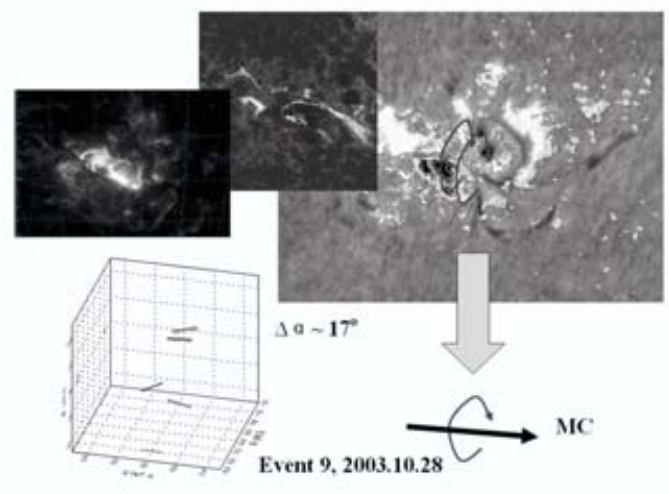

(c)

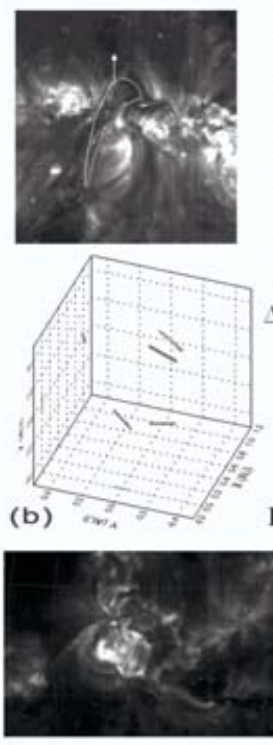

Event 10, 2003.11.18

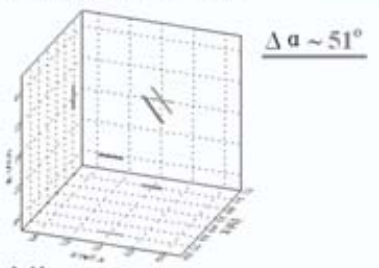

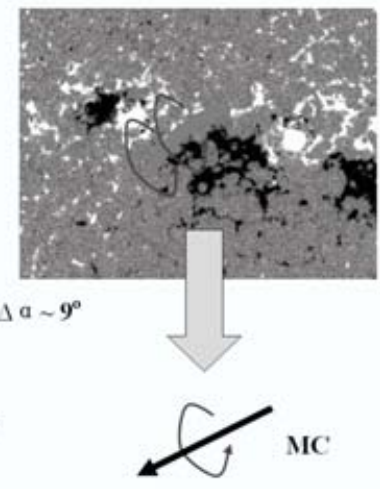

Event 3, 2000.8.9
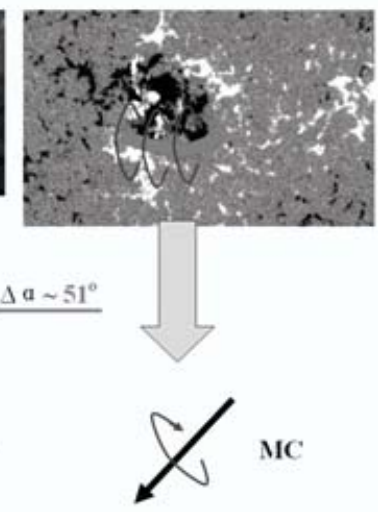

(d)

Figure 2. The solar observations of the four erupted filaments and their comparisons with the interplanetary magnetic clouds.

\section{Discussions}

According to the above analyses, the orientations of magnetic clouds are approximately along the directions of erupted filaments, but not always. This result indicates that using observations of erupted filaments to estimate orientations of magnetic clouds in the interplanetary medium takes a risk. Why is there an exception? We think that there are following three possible reasons: (1) The error in fitting interplanetary MCs by the flux rope model is too large; (2) the ambient solar wind deforms the configuration of magnetic fields inside MCs, and therefore makes their magnetic axes deviate from the initial directions; (3) the axes will rotate when MCs are propagating in the heliosphere. Fig. 1(b) suggests that the variation of intensities of geomagnetic storms is sensitive in $\theta$. Thus, if the orientations of MCs really change when they are ejected out into the interplanetary medium, such change will significantly affects the level of possible geomagnetic storms.

For the four filament-associated MCs, the directions of the ring fields of them are all consistent with the directions of the arcades overlying the erupted filaments. It seems that the rotation of magnetic fields inside clouds can be derived from observations of arcades, i.e., from the polarities of photospheric magnetic fields beside erupted filaments. However, in theory, the ring fields are not necessary consistent with the arcades (refer to Low and Zhang (2002)). Basically, there are two categories of filament: normal type 
and inverse type. For inverse filaments, the ring fields are consistent with the arcades, whereas for normal filaments, it is reversed. According to this theory, above four events are all associated with inverse filaments. Observations suggest that the number of inverse filaments is much larger than that of normal filaments. This is a reason why there is no normal filament in our sample.

To predict intensities of geomagnetic storms several tens hours before their occurrences is difficult. Using observations of erupted filaments and flux rope model to do prediction needs to know not only the orientations $(\theta$ and $\phi)$ of magnetic clouds, but also $B_{0}, R$, and $D$. Thus, much more further work is required.

\section{Conclusions}

(1) For typical MCs, flux rope model can well estimate the intensities of geomagnetic storms. (2) To cause large geomagnetic storms, the favorable orientations of MCs are approximately at $\theta \sim 70^{\circ}$ and $\phi \sim 40^{\circ}$ in GSE coordinates. (3) The orientations of most MCs are roughly along the directions of associated erupted filaments, but not exactly. Occasionally, deviation is very large. (4) The ring fields of magnetic clouds are consistent with the arcades overlying the filaments. It is suggested that the erupted filaments are dominated by inverse type.

\section{Acknowledgements}

We acknowledge the use of the data from the SOHO, Trace and ACE spacecraft. This work is supported by the Chinese Academy of Sciences (KZCX2-SW-136), the National Natural Science Foundation of China (40404014, 40336052, 40336053), and the State Ministry of Science and Technology of China (G2000078405).

\section{References}

V. Bothmer and R. Schwenn 1994, Space Sci. Rev. 70, 215.

L.F. Burlaga 1988, J. Geophys. Res. 93, 7217.

R.K. Burton, R.L. McPherron, and C.T. Russell 1975, J. Geophys. Res. 80, 4204.

W.D. Gonzalez, A. Dal Lago, A.L.C. de Gonzalez, L.E.A. Vieira, and B.T. Tsurutani 2004, J. Atmos. Solar-Terres. Phys. 66, 161-165.

W.D. Gonzalez, J.A. Joselyn, Y. Kamide, H.W. Kroehl, G. Rostoker, B.T. Tsurutani, and V.M. Vasyliunas 1994, J. Geophys. Res. 99, 5771.

W.D. Gonzalez, B.T. Tsurutani, A.L.C. Gonzalez, E.J. Smith, F. Tang, and S.I. Akasofu 1989, J. Geophys. Res. 94, 8835.

J.T., Gosling, D.J. McComas, J.L. Phillips, and S.J. Bame 1991, J. Geophys. Res. 96, 731.

R.P. Kane 2004, J. Geophys. Res. submitted.

A. Kumar and D.M. Rust 1996, J. Geophys. Res. 101, 15667.

B.C. Low and M. Zhang 2002, Astrophys. J 564, L53-L56.

K. Marubashi 1997, In: N. Crooker, J. A. Joselyn, and J. Feynman (eds.): Coronal Mass Ejections. pp. $147-156$.

A.H. McAllister, S.F. Martin, N.U. Crooker, R.P. Lepping, and R.J. Fitzenreiter 2001, J. Geophys. Res. 106(A12), 29185-29194.

N.R. Sheeley, Jr., R.A. Howard, M.J. Koomen, D.J. Michels, R. Schwenn, K.H. Muhlhauser, and H. Rosenbauer 1985, J. Geophys. Res. 90(A1), 163.

D. Vassiliadis, A.J. Klimas, J.A. Valdivia, and D.N. Baker 1999, J. Geophys. Res. 104, 24957.

Y. Wang, C.L. Shen, S. Wang, and P.Z. Ye 2003, Geophys. Res. Lett. 30(20), 2039.

V.B. Yurchyshyn, H. Wang, P.R. Goode, and Y. Deng 2001, Astrophys. J. 563, 381-388.

X.P. Zhao 2002, In: H. Wang and R. Xu (eds.). Proceedings of the COSPAR Colloquium held in the NAOC in Beijing, China. p. 209. 


\section{Discussion}

SCHMIEDER: 1. About the event of October 28, 2003, how do you identify the inversion line where the filament is located? Looking at Trace + MDI movies, the filament is over an inversion line more on the west part of your image between the main leading negative spots and the the following positive spots.

2. You are considering the direction of the arcades over the filaments and the filaments themselves.

WANG: 1. The identification of Oct. 28, 2003 event perhaps was not correct in our work. I will check it again.

2. My consideration is the correlation between the magnetic clouds and the filaments and the arcades overlying the filaments. Generally, magnetic clouds are formed by the ejected arcades overlying the filaments, so there should be a close correlation between them. My aim is to find them for predicting the properties of magnetic clouds, interplanetary space and potential geomagnetic storms intensity.

ZHukOv: A comment concerning the discussion about what we see- filament or overlying arcade. In general, the filament material is extremely rarely observed in situ, so what we see as a magnetic cloud in the solar wind corresponds rather to the arcade overlying the filament.

WANG: Yes, maybe I did not clarify clearly in my talk that magnetic clouds are formed by the arcades (the blue curves in figures) overlying filaments (the red line in figure).

JingxiU WANG: Comments: What he referred to about the direction of magnetic lines of force in the magnetic cloud (blue arrows in the figures) is the overall fields in magnetic arcades of the filaments, not the fields inside in the filaments. I agree with the comments made by Schmieder and Zhukov.

WANG: Yes, I mean that whether the rotation of magnetic field inside the magnetic cloud can be predicted by the arcades overlying the associated filaments. The results suggest the direction of the arcades overlying filaments are not always consistent with the rotation of magnetic field of magnetic clouds.

Schwenn: Do "reverse" and "inverse" topologies both exist? Is there bservational evidence?

WANG: I believe the two types do both exist. In the events studied in my work, there is no observational evidence. It is just our supposition. I will try to find evidence in further work.

Delaboudiniere: What is the direction of the magnetic field in, in 1 . the magnetic cloud?, 2. in the filament?, 3. in the arcade? The latter two are perpendicular. Which is parallel to which?

WANG: According to our result, the direction of the axial magnetic field in the magnetic cloud is (anti-) parallel to the direction of the filament, but not always. Perhaps the magnetic cloud will rotate when it is propagating in the heliosphere, so the orientation of the magnetic cloud is not always parallel to the direction of filament. The arcades overlying filaments form the magnetic cloud. But the rotation of the magnetic field in magentic cloud can not be determined by only the polarities of the photospheric field beside the filament, because there are two types of filament: normal and inverse. 